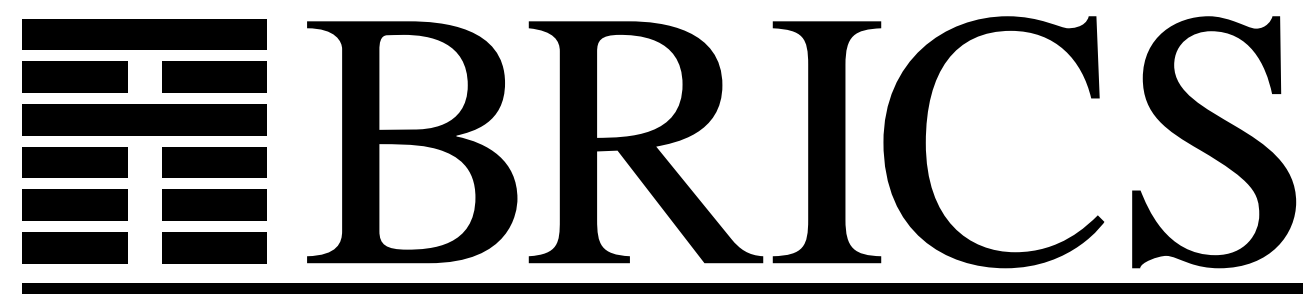

Basic Research in Computer Science

\title{
Detachments Preserving Local Edge-Connectivity of Graphs
}

Tibor Jordán

Zoltán Szigeti

RS-99-35 
Copyright (c) 1999, $\quad$ Tibor Jordán \& Zoltán Szigeti.

BRICS, Department of Computer Science

University of Aarhus. All rights reserved.

Reproduction of all or part of this work is permitted for educational or research use on condition that this copyright notice is included in any copy.

See back inner page for a list of recent BRICS Report Series publications. Copies may be obtained by contacting:

\author{
BRICS \\ Department of Computer Science \\ University of Aarhus \\ Ny Munkegade, building 540 \\ DK-8000 Aarhus C \\ Denmark \\ Telephone: +4589423360 \\ Telefax: $\quad+4589423255$ \\ Internet: BRICS@brics.dk
}

BRICS publications are in general accessible through the World Wide Web and anonymous FTP through these URLs:

http://www.brics.dk

ftp: / / ftp.brics.dk

This document in subdirectory RS/99/35/ 


\title{
Detachments preserving local edge-connectivity of graphs
}

\author{
Tibor Jordán * Zoltán Szigeti ${ }^{\dagger}$
}

November 9, 1999

\begin{abstract}
Let $G=(V+s, E)$ be a graph and let $\mathcal{S}=\left(d_{1}, \ldots, d_{p}\right)$ be a set of positive integers with $\sum d_{j}=d(s)$. An $\mathcal{S}$-detachment splits $s$ into a set of $p$ independent vertices $s_{1}, \ldots, s_{p}$ with $d\left(s_{j}\right)=d_{j}, 1 \leq j \leq p$. Given a requirement function $r(u, v)$ on pairs of vertices of $V$, an $\mathcal{S}$-detachment is called $r$-admissible if the detached graph $G^{\prime}$ satisfies $\lambda_{G^{\prime}}(x, y) \geq r(x, y)$ for every pair $x, y \in V$. Here $\lambda_{H}(u, v)$ denotes the local edge-connectivity between $u$ and $v$ in graph $H$.

We prove that an $r$-admissible $\mathcal{S}$-detachment exists if and only if (a) $\lambda_{G}(x, y) \geq r(x, y)$, and (b) $\lambda_{G-s}(x, y) \geq r(x, y)-\sum\left\lfloor d_{j} / 2\right\rfloor$ hold for every $x, y \in V$.

The special case of this characterization when $r(x, y)=\lambda_{G}(x, y)$ for each pair in $V$ was conjectured by B. Fleiner. Our result is a common generalization of a theorem of W. Mader on edge splittings preserving local edge-connectivity and a result of B. Fleiner on detachments preserving global edge-connectivity. Other corollaries include previous results of L. Lovász and C.J.St.A. Nash-Williams on edge splittings and detachments, respectively. As a new application, we extend a theorem of A. Frank on local edge-connectivity augmentation to the case when stars of given degrees are added.
\end{abstract}

\section{Introduction}

Given a graph $G=(V+s, E)$ with a designated vertex $s$, a degree specification for $s$ is a sequence $\mathcal{S}=\left(d_{1}, \ldots, d_{p}\right)$ of positive integers with $\sum_{j=1}^{p} d_{j}=d(s)$. An $\mathcal{S}$-detachment of $s$ in $G$ is created by replacing $s$ by a set $s_{1}, \ldots, s_{p}$ of independent vertices and distributing the edges incident to $s$ among them in such a way that $d\left(s_{i}\right)=d_{i}(1 \leq i \leq p)$ holds in the new graph. All the other ends of the edges in $G$ remain the same. This operation may decrease (but

*BRICS (Basic Research in Computer Science, Centre of the Danish National Research Foundation), Department of Computer Science, University of Aarhus, Ny Munkegade, building 540, DK-8000 Aarhus, Denmark. e-mail: jordan@daimi.au.dk

${ }^{\dagger}$ Equipe Combinatoire, Université Paris VI, 4, place Jussieu, 75252 Paris, France. e-mail: szigeti@ecp6. jussieu.fr 
cannot increase) the local edge-connectivity between pairs of vertices in $V$. (For a survey on detachments see [7].)

The main result of this paper (Theorem 3.1) gives a necessary and sufficient condition for the existence of an $\mathcal{S}$-detachment of $s$ which satisfies given local edge-connectivity requirements. For a given a requirement function $r: V \times V \rightarrow Z_{+}$, an $\mathcal{S}$-detachment is called $r$-admissible if the detached graph $G^{\prime}$ satisfies $\lambda_{G^{\prime}}(x, y) \geq r(x, y)$ for every pair $x, y \in V$. (We use $\lambda_{H}(u, v)$ to denote local edge-connectivity between $u$ and $v$ in graph $H$, that is, the size of a minimum edge cut separating $u$ and $v$ in $H$.) We characterize those graphs for which an $r$-admissible $\mathcal{S}$ detachment exists. The proof leads to a polynomial algorithm which finds such a detachment if it exists.

Detachments are strongly related to (and generalize) splitting off. Splitting off two edges $s u, s v$ in a graph means replacing $s u, s v$ by a new edge $u v$. If $u=v$ then the resulting loop is deleted. This operation is a well-known and useful tool in proving theorems and designing algorithms for connectivity problems, see e.g. [3, 4, 6]. Subdividing the split edge $u v$ by a new vertex $t$ does not change the local edge-connectivities. Thus a splitting off operation corresponds to a $(2, d(s)-2)$-detachment of $s$ in this sense. Also, the special case of our problem, when $d(s)$ is even, $\mathcal{S}=(2,2, \ldots, 2)$, and $r(x, y)=\lambda_{G}(x, y)$ for each pair $x, y \in V$, corresponds to the problem of finding a 'complete splitting' of the edges incident to $s$ preserving all the local edgeconnectivities within $V$. A celebrated result of W. Mader [6] shows that such a complete splitting always exists (provided there is no cut-edge incident to $s$ ), see Theorem 2.5. This implies that an $r$-admissible $(2,2, \ldots, 2)$-detachment exists for every requirement function $r$ satisfying $r(u, v) \leq$ $\lambda_{G}(x, y)$ for $x, y \in V$.

While such detachments always exist for every 'reasonable' $r$, allowing vertices with degree more than 2 (in particular, allowing odd degrees) in $\mathcal{S}$ gives rise to a new necessary condition. We shall prove that an $r$-admissible $\mathcal{S}$-detachment of $s$ exists if and only if (a) $\lambda_{G}(x, y) \geq r(x, y)$, and (b) $\lambda_{G-s}(x, y) \geq r(x, y)-\sum_{j=1}^{p}\left\lfloor d_{j} / 2\right\rfloor$ hold for every $x, y \in V$.

The special case of this characterization when $r(x, y)=\lambda_{G}(x, y)$ for each pair in $V$ was conjectured by B. Fleiner [1]. Our result is a common generalization of the above mentioned theorem of Mader on edge splittings preserving local edge-connectivity and a result of Fleiner [1] on detachments preserving global edge-connectivity. The latter corresponds to the case when $r(x, y) \equiv k \geq 2$ for each pair $x, y \in V$.

Other corollaries include a theorem of L. Lovász [5] on edge splittings preserving global edge-connectivity and a result of C.J.St.A. Nash-Williams [8] characterizing those graphs $G$ and those lists of degree specifications for which simultaneously detaching the vertices of $G$ preserves global edge-connectivity (see Theorem 6.2). Lovász' theorem follows directly from Theorem 2.5, while Nash-Williams' result can be deduced easily from Fleiner's result [1]. As a new application, we solve a graph augmentation problem where stars of given degrees have to be attached to a given graph in order to meet given local edge-connectivity requirements. This extends a theorem of A. Frank [3] on augmenting the local edge-connectivities by adding a smallest set of new edges.

The organization of the paper is the following. Further definitions and preliminary results are given in Section 2. The proof of the main result starts in Section 3, where we reduce the problem to the case when $\mathcal{S}=(3,3, \ldots, 3)$. Section 4 discusses the method of 'tight set contraction'. 
This method was used previously by Mader and Frank and turned out to be an important step in proving Theorem 2.5. Here we prove several new properties of this operation. This will enable us to handle the remaining case and complete the proof in Section 5. Applications, corollaries, and extensions are discussed in Sections 6 and 7 along with some algorithmic remarks.

\section{Definitions and Preliminaries}

Graphs in this paper are undirected and loopless but may contain multiple edges. Let $G=(V, E)$ be a graph. For $X, Y \subseteq V$ we use $d(X, Y)$ to denote the number of edges from $X-Y$ to $Y-X$. The degree $d(X, V-X)$ of a set $X$ is denoted by $d(X)$. A singleton set $\{v\}$ is simply denoted by $v$. Thus $d(v)$ is the degree of vertex $v \in V$. The symbols $\subseteq$ and $\subset$ denote set containment and proper set containment respectively. Two sets $X, Y$ are said to be intersecting if $X \cap Y, X-Y, Y-X$ are all non-empty. $G$ is $k$-edge-connected if $d(X) \geq k$ holds for every $\emptyset \neq X \subset V$.

The degree function of a graph satisfies the following two well-known equalities.

Proposition 2.1 Let $H=(V, E)$ be a graph. For arbitrary subsets $X, Y \subseteq V$,

$$
\begin{gathered}
d(X)+d(Y)=d(X \cap Y)+d(X \cup Y)+2 d(X, Y), \\
d(X)+d(Y)=d(X-Y)+d(Y-X)+2 d(X \cap Y, V-(X \cup Y)) .
\end{gathered}
$$

Let $G=(V+s, E)$ be a graph and let $r: V \times V \rightarrow Z_{+}$be a requirement function. We define $R(X):=\max \{r(u, v): u \in X, v \in V-X\}$. Clearly, $R(X)=R(V-X)$. Let $s(X):=d(X)-R(X)$. The following property was verified by Frank.

Proposition 2.2 [3, Proposition 5.4] For any two subsets $X, Y \subseteq V$ at least one of the following inequalities holds:

$$
\begin{aligned}
& R(X)+R(Y) \leq R(X \cap Y)+R(X \cup Y), \\
& R(X)+R(Y) \leq R(X-Y)+R(Y-X) .
\end{aligned}
$$

If $X \cup Y=V$ then (4) always holds (with equality).

Propositions 2.1 and 2.2 imply:

Proposition 2.3 For any two subsets $X, Y \subseteq V$ at least one of the following inequalities holds:

$$
\begin{gathered}
s(X)+s(Y) \geq s(X \cap Y)+s(X \cup Y)+2 d(X, Y), \\
s(X)+s(Y) \geq s(X-Y)+s(Y-X)+2 d(X \cap Y, V-(X \cup Y)) .
\end{gathered}
$$

If $X \cup Y=V$ then (6) always holds (with equality). 
Let $s u, s v, s z$ be distinct edges in $G=(V+s, E)$. The operation 2-split deletes the edges $s u, s v$ and adds a new vertex $t$ and two new edges $t u, t v$. Similarly, operation 3-split deletes $s u, s v, s z$ and adds a new vertex $t$ and three new edges $t u, t v, t z$. By definition, both of these operations are special detachments of $s$. Let $r: V \times V \rightarrow Z_{+}$be a requirement function. We say that a 2-split or 3-split is $r$-admissible if the graph $G^{\prime}$ obtained by the split satisfies $\lambda_{G^{\prime}}(u, v) \geq$ $r(u, v)$ for every pair $u, v \in V$. A requirement function is proper for $G$ if $\lambda_{G}(u, v) \geq r(u, v)$ holds for each pair $u, v \in V$. Without loss of generality we shall assume that requirement functions are symmetric, that is, $r(u, v)=r(v, u)$ holds for each pair.

Given $G=(V+s, E)$ and a proper requirement function $r$, we call a set $\emptyset \neq X \subset V$ tight (dangerous) if $s(X)=0(s(X) \leq 1$, respectively). A set with $s(X) \leq 2$ is called bad.

Claim 2.4 A pair su, sv is an r-admissible 2-split if and only if there is no dangerous set $X$ containing $u$ and $v$. A triple su, sv, sz is an $r$-admissible 3-split if and only if (a) there is no tight set $X$ with $|X \cap\{u, v, z\}| \geq 2$ and (b) there is no bad set containing $u, v$ and $z$.

Proof: We prove the claim for 3 -splits. The case of 2-splits is similar (but simpler). Suppose $s u, s v, s z$ is not admissible. This means $\lambda_{G^{\prime}}(x, y)<r(x, y)$ for some pair $x, y \in V$ in the resulting graph $G^{\prime}=\left(V+s+t, E^{\prime}\right)$, where $t$ denotes the new vertex of degree 3 created by the 3 -split. We also have $\lambda_{G}(x, y) \geq r(x, y)$, since $r$ is proper, and $d^{\prime}(Y)<R(Y)$ for some $Y \subset V+t$, by Menger's theorem. Since $d^{\prime}(Z)=d(Z)$ for $t \notin Z$, we must have $t \in Y$. Let $X=Y-t$ and let $\alpha=|X \cap\{u, v, z\}|$. We have $R(X)>d^{\prime}(X)=d(X)-\alpha+(3-\alpha) \geq R(X)-2 \alpha+3$, showing $\alpha \in\{2,3\}$. If $\alpha=3$ then $s(X) \leq 2$ follows. Thus $X$ is a bad set in $G$ containing $u, v, z$. If $\alpha=2$ then $X$ is tight. The other direction is easy to verify by a similar argument.

The counterpart of Claim 2.4 for global edge-connectivity (that is, when $r(u, v) \equiv k \geq 2$ ) appeared in [1]. We remark here that (extensions of) some other basic observations from [1] will also be used later on. Our proof, however, is substantially different from that of [1] in several aspects.

Mader proved the following deep result (see also [2]). To follow our terminology, let us define a requirement function $r_{\lambda}$ by setting $r_{\lambda}(u, v)=\lambda_{G}(u, v)$ for each pair $u, v \in V$.

Theorem 2.5 [6] Let $G=(V+s, E)$ be a graph with $d(s) \neq 3$ such that there is no cut-edge incident to $s$. Then there is an $r_{\lambda}$-admissible 2-split of edges incident to $s$.

\section{Detachments via admissible 2-splits}

In this section we formulate our main result on the existence of an $r$-admissible $\mathcal{S}$-detachment in a graph $G=(V+s, E)$ and give the first part of the proof, where we reduce the problem to the case where $\mathcal{S}=(3,3, \ldots, 3)$. For convenience, we make the following assumptions: (a) $d_{i} \geq 2$, $i=1, \ldots, p$, (b) $G$ is 2-edge-connected. In Section 6 we shall extend our result to the case when these assumptions are dropped. 
Theorem 3.1 Let $r$ be a requirement function for $G=(V+s, E)$ and suppose that $G$ is 2-edgeconnected and $r(u, v) \geq 2$ for each pair $u, v \in V$. Let $\mathcal{S}=\left(d_{1}, \ldots, d_{p}\right)$ be a degree specification for $s$ with $d_{i} \geq 2, i=1, \ldots, p$. Then there exists an $r$-admissible $\mathcal{S}$-detachment of $s$ if and only if $r$ is proper and

$$
\lambda_{G-s}(u, v) \geq r(u, v)-\sum_{i=1}^{p}\left\lfloor d_{i} / 2\right\rfloor
$$

holds for every pair $u, v \in V$ of vertices.

Proof: Suppose that $G^{\prime}$ is obtained from $G$ by an $r$-admissible $\left(d_{1}, \ldots, d_{p}\right)$-detachment. Since $\lambda_{G}(u, v) \geq \lambda_{G^{\prime}}(u, v)$ for each pair $u, v$, the requirement function must be proper. Let us define $D:=\sum_{i=1}^{p}\left\lfloor d_{i} / 2\right\rfloor$. To prove (7) take $\lambda_{G^{\prime}}(u, v)$ pairwise edge-disjoint paths $P_{1}, \ldots, P_{l}(l=$ $\left.\lambda_{G^{\prime}}(u, v)\right)$ in $G^{\prime}$. Since the union of these paths uses an even number of edges from the star of each new vertex $s_{i}(i=1, \ldots, p)$, it follows that at most $D$ paths may go through the set $\left\{s_{1}, \ldots, s_{p}\right\}$. Therefore at least $l-D$ paths lie entirely in $G^{\prime}-\left\{s_{1}, \ldots, s_{p}\right\}=G-s$. This gives $\lambda_{G-s}(u, v) \geq l-D=\lambda_{G^{\prime}}(u, v)-D \geq r(u, v)-D$, as required.

$¿$ From now on we prove that if (7) holds and $r$ is proper then an $r$-admissible $\mathcal{S}$-detachment exists in $G=(V+s, E)$. We start by converting (7) into a more convenient form. Let $d^{*}$ denote the degree function of $G-s$.

Claim 3.2 Condition (7) holds in $G$ if and only if

$$
d^{*}(X) \geq R(X)-D
$$

for every $\emptyset \neq X \subset V$. Furthermore, if (8) holds, we have $d(s, X) \leq s(X)+D$ for every proper subset $X$ of $V$.

Proof: By Menger's theorem having at least $r(u, v)$ edge-disjoint paths between each pair of vertices $u, v \in V$ is equivalent to satisfying $d(X) \geq R(X)$ for every $\emptyset \neq X \subset V$. This implies the first part of the claim. To see the second part observe that $d(s, X)=d(X)-d^{*}(X) \leq$ $d(X)-(R(X)-D)=s(X)+D$ by the definition of $s(X)$ and by (8).

By Claim 3.2 we may suppose $G=(V+s, E)$ satisfies (8) with respect to $\mathcal{S}$ and $r$. We show the existence of the desired detachment by induction on $d(s)$. We may assume $p \geq 2$, since $G$ itself is an $r$-admissible $\mathcal{S}$-detachment otherwise. Thus, since $d_{i} \geq 2$ for $1 \leq i \leq p$, we may also assume $d(s) \geq 4$ and $D \geq 2$. The induction step is based on the following claim.

Suppose that we execute an $r$-admissible 2-split (if $d_{1} \geq 4$ or $d_{1}=2$ ) or a 3-split (if $d_{1}=3$ ) in $G=(V+s, E)$ and let $G^{\prime}=\left(V+t+s, E^{\prime}\right)$ denote the resulting graph, where $t$ is the vertex of degree two or three 'split off' from $s$. The reduced requirement function $r^{\prime}$ in $G^{\prime}$ is defined by $r^{\prime}(u, v)=r(u, v)$ for every pair $u, v \in V$ and $r^{\prime}(t, v)=2$ for every $v \in V$. The reduced degree specification $\mathcal{S}^{\prime}$ of $s$ in $G^{\prime}$ is obtained by decreasing $d_{1}$ appropriately: for a 2split we set $\mathcal{S}^{\prime}=\left(d_{1}-2, \ldots, d_{p}\right)$ (if $d_{1} \geq 4$ ) or $\mathcal{S}^{\prime}=\left(d_{2}, \ldots, d_{p}\right)$ (if $d_{1}=2$ ). For a 3 -split we set $\mathcal{S}^{\prime}=\left(d_{2}, \ldots, d_{p}\right)$ (if $d_{1}=3$ ). Observe that $G^{\prime}$ is 2-edge-connected, $r^{\prime} \geq 2$, and the reduced requirement function is proper in $G^{\prime}$, since the split is $r$-admissible. 
Claim 3.3 Let $\mathcal{S}=\left(d_{1}, \ldots, d_{p}\right)$ be a degree specification for $s$ in $G=(V+s, E)$. Suppose that $G$ has an $r$-admissible 2-split (in the case when $d_{1} \geq 4$ or $d_{1}=2$ ) or an $r$-admissible 3-split (when $\left.d_{1}=3\right)$ at $s$ for which the resulting graph $G^{\prime}=\left(V+t+s, E^{\prime}\right)$ has an $r^{\prime}$-admissible $\mathcal{S}^{\prime}$-detachment, where $r^{\prime}$ and $\mathcal{S}^{\prime}$ are the reduced requirements and reduced degree specification, respectively. Then $G$ has an r-admissible $\mathcal{S}$-detachment.

Proof: The proof is easily seen by observing that an $r$-admissible $\mathcal{S}$-detachment in $G$ can be obtained from the $r^{\prime}$-admissible $\mathcal{S}^{\prime}$-detachment of $G^{\prime}$ by either identifying vertex $t$ and the appropriate vertex of $G^{\prime}$ (when $d_{1} \geq 4$ ), or defining the detachment of $s$ in $G$ as the union of the 2 -split (or 3-split) and the $r^{\prime}$-admissible $\mathcal{S}^{\prime}$-detachment of $s$ in $G^{\prime}$ (when $d_{1}=2$ or $d_{1}=3$ ).

Thus, to make the induction work, it is enough to find an $r$-admissible 2-split (or 3-split) for which the resulting graph $G^{\prime}$ satisfies (8) with respect to the reduced requirement function and the reduced degree specification. An $r$-admissible 2-split or 3-split is called feasible if (8) holds in $G^{\prime}$.

By reducing the degree specification as above we get $D^{\prime}=D-1$, where $D^{\prime}$ is the corresponding value in $G^{\prime}$. Therefore (8) holds in $G^{\prime}$ if and only if $d_{G^{\prime}-s}(X) \geq R^{\prime}(X)-D+1$ for every $\emptyset \neq X \subset V+t$. Notice that for the reduced $r^{\prime}$ and $R^{\prime}$ we have that $R^{\prime}(t)=R^{\prime}(V)=2$, $R^{\prime}(X)=R(X)$ for $\emptyset \neq X \subset V$, and $R^{\prime}(X)=R(X-t)$ otherwise.

Let $\mathcal{B}^{\prime}=\left\{X \subset V: d^{*}(X)=R(X)-D\right\}$ denote those subsets of $V$ which satisfy (8) with equality. Let $\mathcal{B}$ denote the minimal members of $\mathcal{B}^{\prime}$. Sets in $\mathcal{B}$ are called cores.

Claim 3.4 (a) An r-admissible 2-split su, sv is feasible if and only if $|X \cap\{u, v\}| \geq 1$ for every $X \in \mathcal{B}^{\prime}$.

(b) An r-admissible 3-split su, sv, sz is feasible if and only if $|X \cap\{u, v, z\}| \geq 1$ for every $X \in \mathcal{B}^{\prime}$.

Proof: We prove (a) and (b) simultaneously. Let $T=\{u, v\}(T=\{u, v, z\})$ for the $r$-admissible 2 -split (3-split, respectively). Notice that $u, v, z$ are not necessarily distinct. For every proper subset $Y$ of $V$ we have $R^{\prime}(Y)=R(Y)$ and $d_{G^{\prime}-s}(Y)=d^{*}(Y)+|Y \cap T|$. Thus, since $D^{\prime}=D-1$, $G^{\prime}$ satisfies (8) with respect to proper subsets of $V$ if and only if $|X \cap T| \geq 1$ for every $X \in \mathcal{B}^{\prime}$. Condition (8) is trivially met for $t$ and $V$.

Now consider a set $W$ with $t \in W$ and with $Y=W \cap V$ non-empty. For such $W$ we have $R^{\prime}(W)=R(Y)$ and $d_{G^{\prime}-s}(W)=d^{*}(Y)+|(V-Y) \cap T|$. Thus $G^{\prime}$ satisfies (8) with respect to sets $W$ of this type if and only if $|(V-X) \cap T| \geq 1$ for every $X \in \mathcal{B}^{\prime}$. Since $R$ and $d^{*}$ are symmetric in $V$, this is equivalent to saying $|X \cap T| \geq 1$ for every $X \in \mathcal{B}^{\prime}$. This proves the claim.

Lemma 3.5 $|\mathcal{B}| \in\{0,2,3\}$ and the sets in $\mathcal{B}$ are pairwise disjoint. Furthermore, if $|\mathcal{B}|=3$ then $\mathcal{S}=(3,3, \ldots, 3), d(s)=3 D$, and for each $B_{i} \in \mathcal{B}(i=1,2,3)$ we have $d\left(s, B_{i}\right)=D$ and $B_{i}$ is tight. 
Proof: Suppose $\mathcal{B}$ is non-empty. Since $d^{*}$ and $R$ are symmetric in $V,|\mathcal{B}| \geq 2$ follows. Let $B_{1}, B_{2} \in \mathcal{B}$ be intersecting sets. Applying Propositions 2.1 and 2.3 to $B_{1}, B_{2}$ shows that either $B_{1}-B_{2} \in \mathcal{B}^{\prime}$ or $B_{1} \cap B_{2} \in \mathcal{B}^{\prime}$, contradicting the minimality of $B_{1}$. Thus sets in $\mathcal{B}$ are pairwise disjoint. Since $r$ is proper, $d(B) \geq R(B)$ holds and hence $d(s, B) \geq D$ for each $B \in \mathcal{B}$. ¿From the definition of $D$ it is clear that $d(s) \leq 3 D$ and $d(s)=3 D$ if and only if $\mathcal{S}=(3,3, \ldots, 3)$. This implies $|\mathcal{B}| \leq 3$ and that $|\mathcal{B}|=3$ may hold only if $\mathcal{S}=(3,3, \ldots, 3), d(s)=3 D$, and $d\left(s, B_{i}\right)=D$ for each $B_{i} \in \mathcal{B}, i=1,2,3$. The last equality gives $d\left(B_{i}\right)=R\left(B_{i}\right)$, thus each $B_{i}$ is tight.

In the first part of the proof we assume that, without loss of generality, $d_{1} \neq 3$ and show that a feasible and $r$-admissible 2 -split exists. (The remaining case, when $\mathcal{S}=(3,3, \ldots, 3)$, will be dealt with in Section 5.)

Lemma 3.5 shows that in this case we have $|\mathcal{B}| \in\{0,2\}$. Since $d(s) \geq 4, r$ is proper, and there are no cut-edges in $G$, Theorem 2.5 implies that an ( $r_{\lambda}$-admissible and hence) $r$-admissible 2 -split exists. If $\mathcal{B}=\emptyset$ then every $r$-admissible 2 -split is feasible by Claim 3.4 and hence we are done by Claim 3.3. The next lemma shows which 2 -split to choose when $|\mathcal{B}|=2$.

Lemma 3.6 Suppose $G=(V+s, E)$ satisfies (8) and $\mathcal{B}=\left\{B_{1}, B_{2}\right\}$. Then every 2-split $s b_{1}, s b_{2}$ with $b_{i} \in B_{i}(i=1,2)$ is $r$-admissible and feasible.

Proof: By the choice of $b_{1}, b_{2}$ and Claim 3.4(a) it is enough to prove that such a 2-split is $r$ admissible. Let $\alpha=d\left(s, B_{1}\right)$ and $\beta=d\left(s, B_{2}\right)$. Note that $\alpha, \beta \geq D$. Since $B_{1} \in \mathcal{B}$, we have $R\left(B_{1}\right)-D=d^{*}\left(B_{1}\right)=d\left(B_{1}\right)-\alpha$ and hence $s\left(B_{1}\right)=\alpha-D$. Similarly, $s\left(B_{2}\right)=\beta-D$.

For a contradiction suppose, by Claim 2.4, that there exists a dangerous set $X \subset V$ with $b_{1}, b_{2} \in X$. Assuming $B_{1} \cup B_{2} \subseteq X$, from (8) we get $R(X)-D \leq d^{*}(X) \leq d(X)-\alpha-\beta \leq$ $d(X)-2 D \leq R(X)+1-2 D$, contradicting $D \geq 2$. Thus we may assume, without loss of generality, that $B_{1}-X \neq \emptyset$. By the choice of $b_{1}$ and $b_{2}$ we have $X \cap B_{1} \neq \emptyset$ and $X-B_{1} \neq \emptyset$.

Since $B_{1}$ and $B_{2}$ are the only cores, each set in $\mathcal{B}^{\prime}$ includes $B_{1}$ or $B_{2}$. Thus $V-\left(X \cup B_{1}\right) \notin \mathcal{B}^{\prime}$ follows. Hence $R\left(V-\left(X \cup B_{1}\right)\right)-D<d^{*}\left(V-\left(X \cup B_{1}\right)\right)$. Using this and the symmetry of $d^{*}$ and $R$ in $V$, we get $d\left(X \cup B_{1}\right) \geq d^{*}\left(X \cup B_{1}\right)+\alpha+1=d^{*}\left(V-\left(X \cup B_{1}\right)\right)+\alpha+1>$ $R\left(V-\left(X \cup B_{1}\right)\right)-D+\alpha+1=R\left(X \cup B_{1}\right)+\alpha-D+1$ and hence $s\left(X \cup B_{1}\right) \geq \alpha-D+2$ follows.

Now we apply Proposition 2.3 to $X$ and $B_{1}$. If (5) holds and $B_{1} \cup X \neq V$ then we have $1+(\alpha-D) \geq s(X)+s\left(B_{1}\right) \geq s\left(X \cap B_{1}\right)+s\left(X \cup B_{1}\right) \geq \alpha-D+2$, a contradiction. Thus we can assume (6) holds for $X$ and $B_{1}$.

Let $\gamma=d\left(s, X \cap B_{1}\right)$. Since $B_{1}$ is a core, we have $R\left(B_{1}-X\right)-D<d^{*}\left(B_{1}-X\right)$. This implies $d\left(B_{1}-X\right)=d^{*}\left(B_{1}-X\right)+\alpha-\gamma>R\left(B_{1}-X\right)-D+\alpha-\gamma$ and therefore $s\left(B_{1}-X\right) \geq \alpha-D+1-\gamma$. From this (6) yields $1+(\alpha-D) \geq s(X)+s\left(B_{1}\right) \geq$ $s\left(X-B_{1}\right)+s\left(B_{1}-X\right)+2 d\left(s, X \cap B_{1}\right) \geq \alpha-D+1+\gamma$. This is a contradiction, since $\gamma \geq 1$ by the existence of the edge $s b_{1}$.

Thus we have seen that if $d_{i} \neq 3$ for some $1 \leq i \leq p$ then there exists an $r$-admissible and feasible 2-split in $G$. By Claim 3.3 this completes the first part of the proof. 


\section{Contracting tight sets}

In this section we prove that, roughly speaking, contracting non-singleton tight sets does not change the problem. Assuming each tight set is a singleton will be a crucial point in the rest of the main proof given in Section 5.

Let us fix a tight set $T \subset V$ with $|T| \geq 2$ in our graph $G=(V+s, E)$, if exists. Recall that for a tight set $T$ we have $s(T)=d(T)-R(T)=0$ and therefore (in fact, equivalently) there exists a pair $a, b \in V$, separated by $T$, with $r(a, b)=d(T)$. Let $G^{\prime}=\left(V^{\prime}+s, E^{\prime}\right)$ be obtained from $G$ by contracting $T$ into a single vertex $t$. We use $d^{\prime}$ to denote the degree function in $G^{\prime}$ and we define the requirement function $r^{\prime}$ for $G^{\prime}$ as follows: we set $r^{\prime}(u, v)=r(u, v)$ for $u, v \in V^{\prime}-t$ and we set $r(x, t)=r(t, x)=\max \{r(w, x): w \in T\}$ otherwise. It is easy to see that $G^{\prime}$ is 2-edge-connected, $r^{\prime} \geq 2$ is proper for $G^{\prime}$, and $t$ is a singleton tight set in $G^{\prime}$.

The next lemma follows directly from the definition of $r^{\prime}$, without using the fact that $T$ is tight.

Lemma 4.1 Let $X^{\prime} \subset V^{\prime}$ and let $X$ be the corresponding set in $V$ (that is, $X=X^{\prime}$ if $t \notin X^{\prime}$ and $X=\left(X^{\prime}-t\right) \cup T$ otherwise $)$. Then $R(X)=R^{\prime}\left(X^{\prime}\right)$.

Proof: Since $R^{\prime}(R)$ is symmetric in $V^{\prime}$ ( $V$, respectively), we may assume $t \notin X^{\prime}$. In this case $X=X^{\prime}$ and $X \cap T=\emptyset$ hold. First pick $x \in X, y \in V-X$ with $r(x, y)=R(X)$. If $y \notin T$ then $R^{\prime}\left(X^{\prime}\right)=R^{\prime}(X) \geq r^{\prime}(x, y)=r(x, y)=R(X)$ follows. If $y \in T$ then, by the definition of $r^{\prime}$, we have $R^{\prime}\left(X^{\prime}\right)=R^{\prime}(X) \geq r^{\prime}(t, x) \geq r(y, x)=R(X)$. This proves $R^{\prime}\left(X^{\prime}\right) \geq R(X)$.

To see the other direction choose $x^{\prime} \in X^{\prime}, y^{\prime} \in V^{\prime}-X^{\prime}$ with $r^{\prime}\left(x^{\prime}, y^{\prime}\right)=R^{\prime}\left(X^{\prime}\right)$. If $y^{\prime} \neq t$ then $R(X)=R\left(X^{\prime}\right) \geq r\left(x^{\prime}, y^{\prime}\right)=r^{\prime}\left(x^{\prime}, y^{\prime}\right)=R^{\prime}\left(X^{\prime}\right)$ follows. If $y^{\prime}=t$ then $R(X)=R\left(X^{\prime}\right) \geq \max \left\{r\left(w, x^{\prime}\right): w \in T\right\}=r^{\prime}\left(t, x^{\prime}\right)=R^{\prime}\left(X^{\prime}\right)$. This gives $R(X) \geq R^{\prime}\left(X^{\prime}\right)$.

We obtain the following two easy but useful corollaries.

Lemma 4.2 Let su, sv be an r-admissible 2-split in $G$. Then $s u^{\prime}, s v^{\prime}$ is $r^{\prime}$-admissible in $G^{\prime}$.

Proof: Suppose that $s u^{\prime}, s v^{\prime}$ is not $r^{\prime}$-admissible in $G^{\prime}$. Then by Claim 2.4 there exists a dangerous set $X^{\prime} \subset V^{\prime}$ with $s^{\prime}\left(X^{\prime}\right)=d^{\prime}\left(X^{\prime}\right)-R^{\prime}\left(X^{\prime}\right) \leq 1$ and $u^{\prime}, v^{\prime} \in X^{\prime}$. Since $d(X)=d^{\prime}\left(X^{\prime}\right)$ and by Lemma 4.1 we have $R(X)=R^{\prime}\left(X^{\prime}\right)$ for the corresponding set $X$ in $G$, it follows that $X$ is a dangerous set in $G$ which contains $u$ and $v$, a contradiction.

Lemma 4.3 Suppose $G$ satisfies (8). Then $G^{\prime}$ satisfies (8) with respect to $V^{\prime}$ and $r^{\prime}$.

Proof: Suppose (8) fails in $G^{\prime}$ and let $W^{\prime} \subset V^{\prime}$ be a set with $d_{G^{\prime}-s}\left(W^{\prime}\right)<R^{\prime}\left(W^{\prime}\right)-D$. Since $d_{G-s}(W)=d_{G^{\prime}-s}\left(W^{\prime}\right)$ and by Lemma 4.1 we have $R(W)=R^{\prime}\left(W^{\prime}\right)$ for the corresponding set $W$ in $G$, it follows that $d_{G-s}(W)<R(W)-D$, a contradiction.

Mader [6] and Frank [2, Claim 3.2] showed that if a 2-split is $r_{\lambda^{\prime}}$-admissible in $G^{\prime}$ then the corresponding 2-split is $r_{\lambda}$-admissible in $G$. We need a similar fact for 3-splits and arbitrary proper requirement functions $r$. 
Lemma 4.4 Let $s u^{\prime}, s v^{\prime}, s z^{\prime}$ be an $r^{\prime}$-admissible 3-split in $G^{\prime}$. Then the corresponding edges su, sv, sz form an r-admissible 3-split in $G$.

Proof: Suppose that the triple $s u, s v, s z$ is not $r$-admissible in $G$. By Claim 2.4 this implies that in $G$ either (a) there is a tight set $X$ with $|X \cap\{u, v, z\}| \geq 2$ or (b) there is a bad set $M$ containing $u, v$ and $z$.

Let $Y$ be a subset of $V$ with either $Y \cap T=\emptyset$ or $T \subseteq Y$ and let $Y^{\prime}$ be the set corresponding to $Y$ in $G^{\prime}$. Such a set $Y$ clearly satisfies $R^{\prime}\left(Y^{\prime}\right)=R(Y)$ (by Lemma 4.1) and $d^{\prime}\left(Y^{\prime}\right)=$ $d(Y)$. Therefore if $Y$ is tight (bad) in $G$ with respect to $r$ then $Y^{\prime}$ is tight (bad, respectively) in $G^{\prime}$ with respect to $r^{\prime}$. Thus we may assume that $(*)$ for any tight set $X^{\prime}$ (bad set $M^{\prime}$ ) with $\left|X^{\prime} \cap\{u, v, z\}\right| \geq 2\left(\{u, v, z\} \subset M^{\prime}\right)$ the sets $X^{\prime}$ and $T\left(M^{\prime}\right.$ and $T$, respectively) are intersecting.

Consider case (a) first and assume, without loss of generality, that that $u, v \in X$ for a tight set $X$ in $G$. Since $X \cup T$ cannot be tight by $(*)$, we have $s(X \cup T) \geq 1$. We apply Proposition 2.3 to $X$ and $T$. If (5) holds and $X \cup T \neq V$ then, using the fact that $T$ is tight, we get $0+0=s(X)+s(T) \geq s(X \cap T)+s(X \cup T) \geq 0+1$, a contradiction.

Thus (6) holds. In this case we have $0+0=s(X)+s(T) \geq s(X-T)+s(T-X)+$ $2 d(s, X \cap T)$. Therefore $d(s, X \cap T)=0$, so $u, v \in X-T$ follows. We also get $s(X-T)=0$, contradicting $(*)$. This shows case (a) cannot hold.

Suppose now that case (b) holds, that is, there is a bad set $M$ containing $u, v$ and $z$ in $G$. Since $M \cup T$ cannot be bad by (*), we have that either $M \cup T=V$ or $s(M \cup T) \geq 3$. We apply Proposition 2.3 to $M$ and $T$. If (5) holds and $M \cup T \neq V$ then, using the fact that $T$ is tight, we get $2+0 \geq s(M)+s(T) \geq s(M \cap T)+s(M \cup T) \geq 0+3$, a contradiction. Thus (6) holds and we have $2+0 \geq s(M)+s(T) \geq s(M-T)+s(T-M)+2 d(s, M \cap T)$. This shows $d(s, M \cap T) \leq 1$. Moreover, if $d(s, M \cap T)=1$ then $s(M-T)=0$ must hold and hence $M-T$ is a tight set including two vertices from $u, v, z$, contradicting the fact that case (a) does not hold. Thus $d(s, M \cap T)=0$ and $s(M-T) \leq 2$. This contradicts $(*)$.

\section{The case when $\mathcal{S}=(3,3, \ldots, 3)$}

In this section we assume $\mathcal{S}=(3,3, \ldots, 3)$ and show the existence of an $r$-admissible and feasible 3 -split in $G$. This will complete the proof by Claim 3.3. First we consider the case $|\mathcal{B}|=3$. In this case we can avoid tight set contractions. We use the following two claims instead.

Claim 5.1 Let $X$ be a maximal tight set and let $Y$ be tight with $Y-X \neq \emptyset$. Then $d(s, X \cap Y)=$ 0 .

Proof: We may assume $X \cap Y \neq \emptyset$ and apply Proposition 2.3 to $X$ and $Y$. If (5) holds and $X \cup Y \neq V$ then $0+0=s(X)+s(Y) \geq s(X \cap Y)+s(X \cup Y) \geq 0+0$ follows and hence $s(X \cup Y)$ is also tight, contradicting the maximality of $X$. Thus (6) applies. Then $0+0=s(X)+s(Y) \geq s(X-Y)+s(Y-X)+2 d(s, X \cap Y)$, and hence $d(s, X \cap Y)=0$.

Let $s u, s v, s z$ be distinct edges incident to $s$. These three edges (and also the multiset $\{u, v, z\}$ of vertices) is called a legal triple if there is no tight set $X$ with $|X \cap\{u, v, z\}| \geq 2$. 
Claim 5.2 Let $K$ be a tight set and let $L$ be a maximal bad set such that $d(s, K \cap L) \geq 1$ and for a legal triple $u, v, z$ we have $u, v, z \in L$. Then $K \subseteq L$.

Proof: We apply Proposition 2.3 to $K$ and $L$. If (5) holds and $K \cup L \neq V$ then, by the maximality of $L$, we get $0+2 \geq s(K)+s(L) \geq s(K \cap L)+s(K \cup L) \geq 0+3$, a contradiction. Thus (6) applies. Then $0+2=s(K)+s(L) \geq s(K-L)+s(L-K)+2 d(s, K \cap L) \geq 0+0+2$, since $d(s, K \cap L) \geq 1$ by our assumption. This gives $d(s, K \cap L)=1$ and $s(L-K)=0$. Hence at least two edges of the triple must go from $s$ to the tight set $L-K$. This contradicts the fact that the triple is legal.

Lemma 5.3 Let $\mathcal{B}=\left\{B_{1}, B_{2}, B_{3}\right\}$. Then every 3 -split $s b_{1}, s b_{2}$, sb $b_{3}$ with $b_{i} \in B_{i}(i=1,2,3)$ is $r$-admissible and feasible.

Proof: By the choice of $b_{i}(i=1,2,3)$ and Claim 3.4 it is enough to prove that a 3 -split of this kind is $r$-admissible. To see that $s b_{1}, s b_{2}, s b_{3}$ with $b_{i} \in B_{i}(i=1,2,3)$ is $r$-admissible, by Claim 2.4 it is sufficient to show that the triple is legal and there is no bad set $M$ including $b_{1}, b_{2}, b_{3}$.

Suppose that there is a maximal tight set $X$ including $b_{1}$ and $b_{2}$. By Lemma 3.5 each $B_{i}$ is tight. Thus Claim 5.1 implies $\left(B_{1} \cup B_{2}\right) \subseteq X$. Therefore, by Claim 3.2, $D=D+s(X) \geq$ $d(s, X) \geq 2 D$ follows, contradicting the fact that $D \geq 2$. Thus the triple is legal.

Now suppose there is a maximal bad set $M$ including $b_{1}, b_{2}, b_{3}$. Since $M$ includes the endvertices of a legal triple and each $B_{i}$ is tight by Claim 3.5, Claim 5.2 implies $\left(B_{1} \cup B_{2} \cup B_{3}\right) \subseteq M$ and therefore by Claim 3.2 we get $2+D \geq s(M)+D \geq d(s, M) \geq 3 D$, a contradiction.

In the rest of the proof we can assume $|\mathcal{B}| \in\{0,2\}$. In this case we prove the existence of the required 3-split with the help of the results of Section 4. In the following lemmas we shall consider a 2-edge-connected graph $H=(V+s, E)$ with degree specification $\mathcal{S}=\left(d_{1}, \ldots, d_{p}\right)$ for $s$ where

$$
\text { every tight set is a singleton }
$$

with respect to a proper requirement function $r \geq 2$.

Lemma 5.4 Suppose that $H=(V+s, E)$ satisfies (8), (9) and $d(s)>2 D$. Let su, sv be an $r$-admissible 2-split in $H$. Then there exists an edge sz for which su, sv, sz is a legal triple.

Proof: Suppose that some edge $s a$, different from $s u, s v$, does not form a legal triple with $s u$ and $s v$. Since the pair $s u, s v$ is $r$-admissible, by Claim 2.4 there is no tight set containing both $u$ and $v$. Therefore a tight set including $a$ and one of $u$ or $v$ must exist. Now (9) implies that, without loss of generality, $a=u$ and $\{u\}$ is tight. Thus by Claim $3.2 d(s, u) \leq D$ holds and hence there exists an edge $s b$, different from $s v$, with $b \neq u$. If the triple $s u, s v, s b$ is not legal either, then we have $b=v$ and $\{v\}$ is tight. Again, $d(s, v) \leq D$ follows and hence, since $d(s)>2 D$, there exists an edge $s z$ with $z \neq u, v$. This edge $s z$ will do.

In the next lemma we shall improve on Lemma 5.4 and prove that every $r$-admissible 2 -split $s u, s v$ is part of an $r$-admissible 3 -split unless $H$ has a special structure. 
A graph $H^{\prime}=(V+s, E)$ with $d(s)=6$ is called a 2-obstacle (with respect to a given requirement function $r$ ) if there exist two bad sets $M_{1}, M_{2}$ in $H^{\prime}$ such that (i) $M_{1}-M_{2}$ and $M_{2}-M_{1}$ are tight, (ii) $d\left(s, M_{1}-M_{2}\right)=d\left(s, M_{1} \cap M_{2}\right)=d\left(s, M_{2}-M_{1}\right)=2$, and (iii) the 2-split $s u, s v$ is $r$-admissible for $u, v \in M_{1} \cap M_{2}$. It is easy to see that there is no $r$-admissible 3 -split in a 2-obstacle which contains the edges $s u, s v$. On the other hand, an $r$-admissible $(3,3)$ detachment is easy to find in a 2 -obstacle.

Lemma 5.5 Let $H=(V+s, E)$ be a 2-obstacle and suppose that (9) holds. Then $H$ has an $r$-admissible $(3,3)$-detachment.

Proof: It is enough to find an $r$-admissible 3 -split in $H$. Let the (singleton) tight sets $M_{1}-M_{2}$ and $M_{2}-M_{1}$ be vertices $a$ and $b$, respectively. We claim that every triple $s a, s u, s b$ forms an $r$-admissible 3 -split. To see this notice that the triple is legal, since $a, u, b$ are distinct and by (9). Furthermore, a bad set $N$ with $a, u, b \in N$ would have $d(s, N) \geq 5$, contradicting Claim 3.2.

Lemma 5.6 Suppose that $H=(V+s, E)$ satisfies (8), (9), and $d(s)=3 D$. If $H$ is not a 2-obstacle then for every r-admissible 2-split su, sv in $H$ there exists an edge sz such that su, sv, sz form an r-admissible 3-split.

Proof: By Lemma 5.4 there exists an edge $s a$ such that $s u, s v, s a$ is a legal triple. If this triple forms an $r$-admissible 3 -split, we are done. Otherwise by Claim 2.4 there exists a maximal bad set $M$ containing $u, v, a$. Since, by Claim 3.2, we have $d(s, M) \leq s(M)+D \leq 2+D$ and we also have $D \geq 2$, our assumption $d(s)=3 D$ implies that there exists an edge $s b$ with $b \in V-M$. Observe that $u, v, b$ form a legal triple by (9). Thus either $s b$ is the desired edge or there exists a maximal bad set $M^{\prime}$ containing $u, v, b$. Clearly, $M$ and $M^{\prime}$ are intersecting.

We apply Proposition 2.3 to $M$ and $M^{\prime}$. If (5) holds and $M \cup M^{\prime} \neq V$ then we get $2+2 \geq$ $s(M)+s\left(M^{\prime}\right) \geq s\left(M \cap M^{\prime}\right)+s\left(M \cup M^{\prime}\right) \geq 2+3$, a contradiction. Here we used $s\left(M \cap M^{\prime}\right) \geq 2$ (since $s u, s v$ is an $r$-admissible 2-split) and $s\left(M \cup M^{\prime}\right) \geq 3$ (by the maximality of $M$ ).

Thus we can assume that (6) holds. Let $N:=V-\left(M \cup M^{\prime}\right)$. We obtain $2+2 \geq s(M)+$ $s\left(M^{\prime}\right) \geq s\left(M-M^{\prime}\right)+s\left(M^{\prime}-M\right)+2 d\left(N+s, M \cap M^{\prime}\right) \geq 2 d\left(N+s, M \cap M^{\prime}\right)$. The existence of the edges $s u, s v$ implies that the right-hand side is at least 4 . It follows that equality holds everywhere, in particular $s\left(M-M^{\prime}\right)=s\left(M^{\prime}-M\right)=0$ and $d\left(N+s, M \cap M^{\prime}\right)=2$ must hold. Therefore $M-M^{\prime}$ and $M^{\prime}-M$ are both tight and by Claim 3.2 we have $d\left(s, M-M^{\prime}\right), d\left(s, M^{\prime}-\right.$ $M) \leq D$. In this case $d(s, N)=0$ would imply $2 D+2 \geq d(s)=3 D$ and hence $D=2$. Thus $H$ would be a 2-obstacle (by taking $M_{1}=M$ and $M_{2}=M^{\prime}$ in the definition), a contradiction.

This shows $d(s, N) \geq 1$. Let us pick an edge $s c$ with $c \in N$. As above, it can be seen that $u, v, c$ is a legal triple and either $s c$ is the desired edge or there is a maximal bad set $M^{\prime \prime}$ containing $u, v, c$. In the latter case we can apply Proposition 2.3 to $M$ and $M^{\prime \prime}$ and to $M^{\prime}$ and $M^{\prime \prime}$, as above, and conclude that $M-M^{\prime \prime}, M^{\prime \prime}-M, M^{\prime}-M^{\prime \prime}, M^{\prime \prime}-M^{\prime}$ are all tight. Since (9) holds and these bad sets are pairwise distinct and maximal, it follows that $M \cap M^{\prime}=M \cap M^{\prime \prime}=M^{\prime} \cap M^{\prime \prime}$ and $M \cup M^{\prime} \cup M^{\prime \prime}=M \cap M^{\prime} \cap M^{\prime \prime} \cup\{a, b, c\}$.

Claim 5.7 There is an edge in $H$ from a to $M-a$ (and similarly from $b$ to $M^{\prime}-b$ and from $c$ to $\left.M^{\prime \prime}-c\right)$. 
Proof: Suppose that no such edge exists and first consider the case when $R(M)$ is attained on a pair $x, y$ with $x \in M-a$. In this case $R(M-a) \geq R(M)$ and since $d(a) \geq 2$ we conclude that $d(M-a) \leq d(M)-2$ and hence $M-a$ is tight. Since $u, v \in M-a$, this contradicts the fact that $s u, s v$ is an $r$-admissible 2-split. In the other case, when $R(M)=r(a, y)$ for some $y \in V-M$, we have $R(M) \leq R(a) \leq d(a) \leq d(M)-d(M-a) \leq R(M)+2-2=R(M)$, and hence $d(M-a)=2$ must hold. Thus, since $r \geq 2$, we have $R(M-a) \geq 2$ and hence the set $M-a$ is tight. Since $u, v \in M-a$, this contradicts the fact that the 2 -split $s u, s v$ is $r$-admissible. This proves the claim.

The edge from $c$ to $M^{\prime \prime}-c=M \cap M^{\prime}$, which exists by Claim 5.7, implies that $d\left(c, M \cap M^{\prime}\right) \geq$ 1. Together with the edges $s u, s v$ this gives $d\left(N+s, M \cap M^{\prime}\right) \geq 3$, a contradiction. This proves the lemma.

By Claim 3.3 the proof is complete once we can show an $r$-admissible and feasible 3 -split in $G$. If $|\mathcal{B}|=3$ then we are done by Lemma 5.3. Suppose $|\mathcal{B}| \in\{0,2\}$. In this case, starting from $G$, let us contract non-singleton tight sets as long as possible and let $G^{\prime}=\left(V^{\prime}+s, E^{\prime}\right)$ and $r^{\prime}$ denote the resulting graph and requirement function, respectively. By Lemma $4.3 G^{\prime}$ satisfies (8) with respect to $V^{\prime}$ and $r^{\prime}$ and by construction (9) holds. Note that the degree specification for $s$ in $G$ and in $G^{\prime}$ are the same (hence $d^{\prime}(s)=3 D \geq 6$ ).

Let us choose an $r$-admissible 2-split $s u, s v$ in $G$ in such a way that $u$ and $v$ are chosen from distinct cores in the case when $G$ has precisely two cores. By Theorem 2.5 and by Lemma 3.6 this can be done. By Lemma 4.2 the corresponding 2-split $s u^{\prime}, s v^{\prime}$ is $r^{\prime}$-admissible in $G^{\prime}$. Thus by Lemma 5.5 and Lemma 5.6 there exists an $r^{\prime}$-admissible 3-split $s u^{\prime}, s v^{\prime}, s z^{\prime}$ in $G^{\prime}$. Applying Lemma 4.4 we can see that the corresponding 3-split $s u, s v, s z$ is $r$-admissible in $G$. By the choice of the pair $s u, s v$ and by Claim 3.4 this $r$-admissible 3 -split in $G$ is feasible as well. This completes the proof of Theorem 3.1.

\section{Applications and corollaries}

It is easy to see that in Theorem 2.5 it is sufficient to consider 2-edge-connected graphs. Thus Theorem 2.5 follows immediately from Theorem 3.1 by taking $\mathcal{S}=(2,2, \ldots, 2)$ and $r=r_{\lambda}$. Note that in this case condition (7) is void.

The special case of Theorem 3.1, when $r=r_{\lambda}$ and $\mathcal{S}$ is arbitrary, was posed as a conjecture by Fleiner [1]. In the same paper Fleiner proved the following 'global edge-connectivity' version of our result. Let us call a detachment $k$-admissible for some $k \geq 2$ if it is $r$-admissible for the uniform requirements $r(u, v) \equiv k$. This result is also an immediate corollary of Theorem 3.1.

Theorem 6.1 [1] Suppose that $G=(V+s, E)$ is $k$-edge-connected in $V$ (for some $k \geq 2$ ) and let $\mathcal{S}=\left(d_{1}, \ldots, d_{p}\right)$ be a degree specification with $\sum_{j=1}^{p} d_{j}=d(s)$ and $d_{j} \geq 2,1 \leq j \leq p$. There 
exists a $k$-admissible $\mathcal{S}$-detachment of $s$ if and only if $G-s$ is $\gamma$-edge-connected, where

$$
\gamma=k-\sum_{i=1}^{r}\left\lfloor d_{i} / 2\right\rfloor .
$$

Theorem 6.1 extends a theorem of Lovász [5] on edge splittings preserving global edgeconnectivity the same way as Theorem 3.1 extends Theorem 2.5. Another application of Theorem 6.1, which was pointed out by Fleiner [1], is the following theorem of Nash-Williams [8]. Given a collection of degree specifications $\mathcal{S}_{v}$, one for each vertex $v$ of $G$, a simultaneous $\mathcal{S}$-detachment is obtained from $G$ by applying an $\mathcal{S}_{v}$-detachment at $v$, for each vertex $v$, simultaneously.

Theorem 6.2 [8] Let $G=(V, E)$ be a graph and let a degree specification $\mathcal{S}_{v}=\left(d_{1}^{v}, \ldots, d_{p_{v}}^{v}\right)$ be given for each vertex $v \in V$. Then $G$ has a $k$-edge-connected simultaneous $\mathcal{S}$-detachment if and only if $G$ is $k$-edge-connected and $d_{i}^{v} \geq k$ for every $v \in V, 1 \leq i \leq p_{v}$, unless $k$ is odd and either $G$ has a cut-vertex $v$ with $\mathcal{S}_{v}=(k, k)$, or $V=\{u, v\}$ and $\mathcal{S}_{u}=\mathcal{S}_{v}=(k, k)$.

It would be interesting to prove some kind of local edge-connectivity version of Theorem 6.2, using Theorem 3.1.

In what follows we apply Theorem 3.1 to deduce a new result. In local edge-connectivity augmentation problem a graph $G=(V, E)$ and a requirement function $r(u, v), u, v \in V$ are given, and the goal is to find a smallest set $F$ of new edges for which the augmented graph satisfies the requirements, that is, for which in $G^{\prime}=(V, E+F)$ we have $\lambda^{\prime}(u, v) \geq r(u, v)$ for each pair $u, v \in V$. This problem was solved, in terms of a min-max equality and a polynomial algorithm, by Frank [3]. A possible extension of this problem has the same input, but instead of adding edges (or equivalently, attaching degree-two vertices) the goal is to attach stars of some given size. In such a 'star-attachment' the centers of the stars are required to be disjoint from $V$ and from each other and their leaves are incident to $V$.

For convenience, we assume $r(u, v) \geq 2$ for each pair $u, v$. Without this assumption one has to deal with the so-called "marginal components' as well, see [3]. The extension to arbitrary requirements can be done following [3]. We leave these details to the reader.

Let $G=(V, E)$ and a requirement function $r(u, v)$ be given along with a set of integers $d_{1}, \ldots, d_{p}\left(d_{j} \geq 2\right)$. Let $R(X)=\max \{r(u, v): u \in X, v \in V-X\}$ be defined for each proper subset $X$ of $V$ and let $q(X)=R(X)-d(X)$. A component of $G$ is called a marginal component if $q(W) \leq 0$ for every $W \subset C$ and $q(C) \leq 1$ hold. For simplicity, we assume $G$ has no marginal components with respect to $r$. (The extension to arbitrary requirements can be done following [3]. We leave these details to the reader.)

Theorem 6.3 Let a graph $G=(V, E)$ and local edge-connectivity requirements $r(u, v), u, v \in$ $V$ be given such that there is no marginal component in $G$. Then $G$ can be augmented to satisfy the requirements $r(u, v)$ by attaching $p$ stars with degrees $d_{1}, \ldots, d_{p}\left(d_{j} \geq 2,1 \leq j \leq p\right)$ if and only if

$$
\sum_{i=1}^{t} q\left(X_{i}\right) \leq \sum_{j=1}^{p} d_{j}
$$


holds for every subpartition $\left\{X_{1}, \ldots, X_{t}\right\}$ of $V$ and $\lambda_{G}(u, v) \geq r(u, v)-\sum_{j=1}^{p}\left\lfloor d_{j} / 2\right\rfloor$ for every pair $u, v \in V$.

Proof: Let $H$ be a properly augmented graph. By Menger's theorem we must have $d_{H}(X) \geq$ $R(X)=d(X)+q(X)$ for every $X \subset V$. This shows (11) holds. Observe that $H$ can be obtained from $G$ by adding a new vertex $s$, creating a graph $G^{\prime}=\left(V+s, E^{\prime}\right)$ that satisfies all the requirements in $V$ and has $d(s)=\sum_{j=1}^{p} d_{j}$, and then executing an $r$-admissible $\left(d_{1}, \ldots, d_{p}\right)$ detachment at $s$ in $G^{\prime}$. This gives rise to the second necessary condition.

Now we prove these conditions together are sufficient. Let $\beta=\sum_{j=1}^{p} d_{j}$. Since $\sum q\left(X_{i}\right) \leq \beta$ for every subpartition $\left\{X_{1}, \ldots, X_{t}\right\}$, we can apply [3, Lemma 5.6] and conclude that $G$ can be extended to a graph $G^{\prime}=\left(V+s, E^{\prime}\right)$ by adding a new node $s$ and $\beta$ edges between $V$ and $s$ so that no edge incident to $s$ is a cut-edge in $G^{\prime}$ and $\lambda^{\prime}(u, v) \geq r(u, v)$ holds for each pair $u, v \in V$. It is easy to see that we may assume that $G^{\prime}$ is 2-edge-connected and thus we may assume $r \geq 2$. Using the fact that $\lambda_{G}(u, v) \geq r(u, v)-\sum\left\lfloor d_{j} / 2\right\rfloor$ for every pair $u, v \in V$, we can apply Theorem 3.1 and obtain an $r$-admissible $\mathcal{S}$-detachment of $s$. This yields the required augmentation of $G$.

Based on Theorem 6.3 we can easily solve the following optimization problem: given $G$, $r$ and an integer $w$, determine the minimum number of $w$-stars that, by attaching them appropriately, can make $G$ satisfy all the local edge-connectivity requirements $r(u, v)$. If $w=2$ (or equivalently, if $d_{i}=2$ for $1 \leq i \leq p$ in Theorem 6.3), then we are back at Frank's theorem [3].

Theorem 6.4 [3] Given a graph $G=(V, E)$ and local edge-connectivity requirements $r(u, v)$ for each pair $u, v \in V$ such that $G$ has no marginal components, $G$ can be augmented to satisfy the requirements $r(u, v)$ by adding $p$ new edges if and only if $\sum\left(q\left(X_{i}\right)\right) \leq 2 p$ for every subpartition $\left\{X_{1}, \ldots, X_{t}\right\}$ of $V$.

\section{Extensions and algorithmic remarks}

In this section we show some extensions of Theorem 3.1 which apply even if we drop our previous assumptions (a) $d_{i} \geq 2$, and (b) $G$ is 2-edge-connected.

First let $G=(V+s, E)$ and $r$ be given as in Theorem 3.1 but suppose that in the degree specification $\mathcal{S}=\left(d_{1}, \ldots, d_{p}\right)$ we have $d_{i}=1$ for some (say, precisely $b$ ) indices $1 \leq i \leq p$. Condition (7) is clearly necessary to have an $r$-admissible $\mathcal{S}$-detachment in this case, too. Since the degree-one vertices and the edges incident to them, created by some detachment, does not influence the local edge-connectivity values in $V$, it is easy to see that if $G$ has an $r$-admissible $\mathcal{S}$-detachment then we can delete $b$ edges incident to $s$ in $G$ in such a way that $\lambda(u, v) \geq r(u, v)$ is maintained for $u, v \in V$ and (7) is preserved. By Theorem 3.1 we can prove that the converse also holds.

Theorem 7.1 There exists an r-admissible $\mathcal{S}$-detachment in $G=(V+s, E)$ if and only if (7) holds and $\sum_{i=1}^{t} q\left(X_{i}\right) \leq d(s)-b$ for every subpartition $\left\{X_{1}, \ldots, X_{t}\right\}$ of $V$. 
Proof: (sketch) The theorem follows from Theorem 3.1 and the following result of Frank (cf. [3, Lemma 5.6]): if there are no cut-edges incident to $s$ in $G$ then at least $b$ edges incident to $s$ can be deleted in such a way that $r$ remains proper (that is, $d^{\prime}(X) \geq R(X)$ holds in the resulting graph $G^{\prime}$ for every $\left.\emptyset \neq X \subset V\right)$ if and only if $\sum q\left(X_{i}\right) \leq d(s)-b$ for every subpartition $\left\{X_{1}, \ldots, X_{t}\right\}$ of $V$.

In our problem of finding an $r$-admissible $\mathcal{S}$-detachment of $s$, if exists, we may clearly assume that the input graph $G=(V+s, E)$ is connected. In addition, if $r \geq 1$ (that is, connectedness has to be preserved) then we can assume that the cut-edges, if exist, are all incident to $s$. In what follows we sketch how to handle these cut-edges. We shall assume $r \geq 1$ and that $r$ is proper.

Suppose that we have $c$ cut-edges incident to $s$ and let $\hat{G}=(\hat{V}+s, \hat{E})$ denote the graph obtained from $G$ by deleting all the cut-edges incident to $s$ as well as those components of the resulting graph that do not contain $s$. Let $\hat{r}$ be the restriction of $r$ to $\hat{V}$. $\hat{G}$ is either a single vertex $\{s\}$ or is 2-edge-connected. The former case is trivial: either $p=1$ or there is no $r$-admissible detachment. In the latter case we may assume $\hat{r} \geq 2$. It can be seen that an $r$-admissible $\mathcal{S}$ detachment exists in $G$ if and only if $\hat{G}$ has an $\hat{r}$-admissible $\hat{\mathcal{S}}=\left(f_{1}, \ldots, f_{p}\right)$-detachment for some integers $f_{i}$ with $\sum f_{i}=\sum d_{j}-c$ and $1 \leq f_{i} \leq d_{i}, 1 \leq i \leq p$. The existence of such a detachment (and more generally, the problem of finding an $r^{\prime}$-admissible detachment of $s$ with prescribed lower and upper bounds for each number of the possible degree specification) can be solved by Theorems 3.1 and 7.1. We omit the details.

We do not attempt to describe an efficient algorithm for finding an $r$-admissible $\mathcal{S}$-detachment in this paper. We note, however, that our proof is algorithmic and easily implies a polynomial algorithm for this problem as well as for the problem of optimally augmenting a graph by attaching stars of some given size. This follows from the fact that checking whether a 2-split or 3-split is $r$-admissible and feasible is easy by max-flow computations. For the special case of finding $r_{\lambda}$-admissible 2-splits (as in Theorem 2.5) Gabow [4] developed an efficient algorithm.

\section{References}

[1] B. Fleiner, Detachments of vertices of graphs preserving edge-connectivity, submitted to SIAM J. Discrete Math.

[2] A. Frank, On a theorem of Mader, Discrete Mathematics 101 (1992) 49-57.

[3] A. Frank, Augmenting graphs to meet edge-connectivity requirements, SIAM J. Discrete Math. 5, 25-53 (1992).

[4] H.N. Gabow, Efficient splitting off algorithms for graphs, in Proc. 26th Annual ACM Symposium on the Theory of Computing, ACM, New-York, 1994, pp. 696-705.

[5] L. Lovász, Combinatorial Problems and Exercises, Akadémiai Kiadó, 1979.

[6] W. Mader, A reduction method for edge-connectivity in graphs, Ann. Discrete Math. 3 (1978) 145-164. 
[7] C. St. J. A. Nash-Williams, Detachments of graphs and generalised Euler trails, Surveys in combinatorics 1985, 137-151, London Math. Soc. Lecture Note Ser., 103, Cambridge Univ. Press, 1985.

[8] C. St. J. A. Nash-Williams, Connected detachments of graphs and generalized Euler trails, J. London Math. Soc., Vol. 31, 1985, pp. 17-29. 


\section{Recent BRICS Report Series Publications}

RS-99-35 Tibor Jordán and Zoltán Szigeti. Detachments Preserving Local Edge-Connectivity of Graphs. November 1999. 16 pp.

RS-99-34 Flemming Friche Rodler. Wavelet Based 3D Compression for Very Large Volume Data Supporting Fast Random Access. October 1999. 36 pp.

RS-99-33 Luca Aceto, Zoltán Ésik, and Anna Ingólfsdóttir. The MaxPlus Algebra of the Natural Numbers has no Finite Equational Basis. October 1999. 25 pp. To appear in Theoretical Computer Science.

RS-99-32 Luca Aceto and François Laroussinie. Is your Model Checker on Time? - On the Complexity of Model Checking for Timed Modal Logics. October 1999. 11 pp. Appears in Kutyłowski, Pacholski and Wierzbicki, editors, Mathematical Foundations of Computer Science: 24th International Symposium, MFCS '99 Proceedings, LNCS 1672, 1999, pages 125-136.

RS-99-31 Ulrich Kohlenbach. Foundational and Mathematical Uses of Higher Types. September 1999. 34 pp.

RS-99-30 Luca Aceto, Willem Jan Fokkink, and Chris Verhoef. Structural Operational Semantics. September 1999. 128 pp. To appear in Bergstra, Ponse and Smolka, editors, Handbook of Process Algebra, 1999.

RS-99-29 Søren Riis. A Complexity Gap for Tree-Resolution. September 1999. 33 pp.

RS-99-28 Thomas Troels Hildebrandt. A Fully Abstract Presheaf Semantics of SCCS with Finite Delay. September 1999. 37 pp. To appear in Category Theory and Computer Science: 8th International Conference, CTCS '99 Proceedings, ENTCS, 1999.

RS-99-27 Olivier Danvy and Ulrik P. Schultz. Lambda-Dropping: Transforming Recursive Equations into Programs with Block Structure. September 1999. 57 pp. To appear in the November 2000 issue of Theoretical Computer Science. This revised report supersedes the earlier BRICS report RS-98-54. 